\title{
Global Prym-Torelli theorem for double coverings of elliptic curves
}

\author{
Atsushi Ikeda
}

\begin{abstract}
The Prym variety for a branched double covering of a nonsingular projective curve is defined as a polarized abelian variety. We prove that any double covering of an elliptic curve which has more than four branch points is recovered from its Prym variety.
\end{abstract}

\section{Introduction}

Let $C$ and $C^{\prime}$ be nonsingular projective curves, and let $\phi: C \rightarrow C^{\prime}$ be a double covering branched at $2 n$ points. In [Mum74], the Prym variety $P(\phi)$ for the double covering $\phi$ is defined as a polarized abelian variety of dimension $d=g^{\prime}-1+n$, where $g^{\prime}$ is the genus of $C^{\prime}$. Let $\mathcal{R}=\mathcal{R}_{g^{\prime}, 2 n}$ be the moduli space of such coverings, and let $\mathcal{A}=\mathcal{A}_{d}$ be the moduli space of polarized abelian varieties of dimension $d$. Then the construction of the Prym variety defines the Prym map $P: \mathcal{R} \rightarrow \mathcal{A}$, and the Prym-Torelli problem asks whether the Prym map is injective. If $g^{\prime}=0$, then it is injective by the classical Torelli theorem for hyperelliptic curves. We consider the case $g^{\prime}>0$ and $\operatorname{dim} \mathcal{R} \leqslant \operatorname{dim} \mathcal{A}$, where we note that $\operatorname{dim} \mathcal{R}=3 g^{\prime}-3+2 n$ and $\operatorname{dim} \mathcal{A}=\frac{1}{2}\left(g^{\prime}-1+n\right)\left(g^{\prime}+n\right)$. The generical injectivity for the Prym map has been proved in most cases.

Theorem 1.1. The Prym map is generically injective in the following cases:

(i) (Friedman and Smith [FS82], Kanev [Kan83]) $n=0$ and $\operatorname{dim} \mathcal{R}<\operatorname{dim} \mathcal{A}$

(ii) (Marcucci and Pirola [MP12]) $g^{\prime}>1, n>0$, and $\operatorname{dim} \mathcal{R}<\operatorname{dim} \mathcal{A}-1$

(iii) (Naranjo and Ortega [NO19]) $g^{\prime}>1, n>0$ and $\operatorname{dim} \mathcal{R}=\operatorname{dim} \mathcal{A}-1$

(iv) (Marcucci and Naranjo [MN14]) $g^{\prime}=1, n>0$ and $\operatorname{dim} \mathcal{R} \leqslant \operatorname{dim} \mathcal{A}$.

The Prym varieties for unramified coverings have been intensively studied because they are principally polarized abelian varieties. For ramified coverings, Nagaraj and Ramanan [NR95] proved the above Theorem 1.1(ii) for $n=2$, and then Marcucci and Pirola [MP12] proved it for any $n>0$. When $g^{\prime}>1$ and $\operatorname{dim} \mathcal{R}=\operatorname{dim} \mathcal{A}$, there are only two cases, $\left(g^{\prime}, n\right)=(6,0),(3,2)$. If $\left(g^{\prime}, n\right)=(6,0)$, then the Prym map is generically finite of degree 27 (see [DS81]), and if $\left(g^{\prime}, n\right)=(3,2)$, then it is generically finite of degree 3 (see [NR95, BCV95]).

Although the Prym map is not injective for many cases in Theorem 1.1 (see [Don81, Nar92, NR95, Ver04]), we prove the injectivity when $g^{\prime}=1$. The following is the main result of this paper, which improves Theorem 1.1(iv).

Received 08 January 2019, accepted in final form 21 September 2019.

2010 Mathematics Subject Classification 14H40, $14 \mathrm{C} 34$.

Keywords: Prym varieties, Torelli theorem, Gauss maps.

This journal is (C) Foundation Compositio Mathematica 2020. This article is distributed with Open Access under the terms of the Creative Commons Attribution Non-Commercial License, which permits non-commercial reuse, distribution, and reproduction in any medium, provided that the original work is properly cited. For commercial re-use, please contact the Foundation Compositio Mathematica. 


\section{PRYM-TORELli FOR COVERINGS OF ELLIPTIC CURVES}

Theorem 1.2 (Theorem 3.1). If $g^{\prime}=1$ and $n \geqslant 3$, then the Prym map is injective.

We remark that $n \geqslant 3$ is equivalent to the condition $\operatorname{dim} \mathcal{R} \leqslant \operatorname{dim} \mathcal{A}$ when $g^{\prime}=1$. To prove this theorem, we use the Gauss map for the polarization divisor, which is a standard approach to Torelli problems. Let $\mathcal{L}$ be an ample invertible sheaf which represents the polarization of the Prym variety $P=P(\phi)$. For a member $D \in|\mathcal{L}|$, we consider the Gauss map

$$
\Psi_{D}: D \backslash D_{\text {sing }} \longrightarrow \mathbf{P}^{d-1}=\operatorname{Grass}\left(d-1, H^{0}\left(P, \Omega_{P}^{1}\right)^{\vee}\right) .
$$

It is not difficult to show that there exists a member $D_{0} \in|\mathcal{L}|$ such that the branch locus of $\Psi_{D_{0}}$ recovers the original covering $\phi: C \rightarrow C^{\prime}$, in a way similar to Andreotti's proof [And58] of the Torelli theorem for hyperelliptic curves. The essential part of our proof is to distinguish the special member $D_{0} \in|\mathcal{L}|$. We study the restriction $\left.\Psi_{D}\right|_{\text {Bs }|\mathcal{L}|}: \operatorname{Bs}|\mathcal{L}| \backslash D_{\text {sing }} \rightarrow \mathbf{P}^{d-1}$ of the Gauss map to the base locus of the linear system $|\mathcal{L}|$. Although $\Psi_{D}$ is difficult to compute, the restriction $\left.\Psi_{D}\right|_{\mathrm{Bs}|\mathcal{L}|}$ is rather simple for any member $D \in|\mathcal{L}|$. By using the image of $\left.\Psi_{D}\right|_{\mathrm{Bs}}|\mathcal{L}|$ and the branch locus of $\left.\Psi_{D}\right|_{\mathrm{Bs}|\mathcal{L}|}$, we can specify the member $D_{0} \in|\mathcal{L}|$ which has the desired property.

In Section 2, we summarize some basic properties of bielliptic curves and their Prym varieties. In Section 3, we explain the strategy of the proof of Theorem 1.2 by using the key propositions in Section 6. In Section 4, we explicitly describe the base locus of the linear system of polarization divisors. In Section 5, we show that the restricted Gauss map $\left.\Psi_{D}\right|_{\mathrm{Bs}|\mathcal{L}|}$ is the same map as the restriction of the Gauss map for the theta divisor on the Jacobian variety of $C$. By giving a simple description for $\left.\Psi_{D}\right|_{\text {Bs }|\mathcal{L}|}$, we prove some properties on the branch locus of $\left.\Psi_{D}\right|_{\text {Bs }|\mathcal{L}|}$. In Section 6 , we present key propositions, which are consequences of the results in Section 5.

In this paper, we work over an algebraically closed field $k$ of characteristic different from 2 .

\section{Properties of bielliptic curves and Prym varieties}

Let $C$ be a nonsingular irreducible projective curve of genus $g$ over $k$, and let $\sigma$ be an involution on $C$. In this paper, we call the pair $(C, \sigma)$ a bielliptic curve of genus $g$ if $g>1$ and the quotient $E=C / \sigma$ is a nonsingular curve of genus 1 . We denote the quotient morphism by $\phi: C \rightarrow E$. First, we note the following.

Lemma 2.1 ([Nar92, (3.3)]). Let $(C, \sigma)$ be a bielliptic curve of genus $g$. If $g>3$, then $C$ is not a hyperelliptic curve.

Let $N: J(C) \rightarrow J(E)$ be the norm map of $\phi$, which is a homomorphism on the Jacobian varieties.

Lemma 2.2 ([Mum74]). Let $\phi: C \rightarrow E$ be the covering defined from a bielliptic curve $(C, \sigma)$.

(i) The map $\phi^{*}: \operatorname{Pic}^{0}(E) \rightarrow \operatorname{Pic}^{0}(C)$ is injective.

(ii) The kernel $P$ of the norm map $N: J(C) \rightarrow J(E)$ is reduced and connected.

By Lemma 2.2, the kernel $P$ of the norm map $N$ is an abelian variety of dimension $n=g-1$. Let $P^{\vee}$ be the dual abelian variety of $P$, and let $\lambda_{P}: P \rightarrow P^{\vee}$ be the polarization isogeny which is defined as the restriction of the principal polarization on the Jacobian variety $J(C)$. Then the polarized abelian variety $\left(P, \lambda_{P}\right)$ is called the Prym variety for the covering $\phi: C \rightarrow E$. We denote by $K(P) \subset P$ the kernel of the polarization $\lambda_{P}: P \rightarrow P^{\vee}$. An ample invertible sheaf $\mathcal{L}$ 


\section{A. IKEDA}

on $P$ represents the polarization $\lambda_{P}$ if the polarization isogeny $\lambda_{P}$ is given by

$$
\lambda_{P}: P \longrightarrow P^{\vee}=\operatorname{Pic}^{0}(P), \quad x \longmapsto t_{x}^{*} \mathcal{L} \otimes \mathcal{L}^{\vee},
$$

where $t_{x}: P \rightarrow P$ denotes the translation by $x \in P$.

Lemma 2.3 ([Mum74]). Let $\left(P, \lambda_{P}\right)$ be the Prym variety defined from a bielliptic curve $(C, \sigma)$, and let $\mathcal{L}$ be an ample invertible sheaf which represents $\lambda_{P}$.

(i) We have $K(P)=\phi^{*} J(E)_{2} \subset J(C)$, where $J(E)_{2}$ denotes the set of points of order 2 on $J(E)$.

(ii) $\operatorname{deg} \lambda_{P}=4$ and $h^{0}(P, \mathcal{L})=2$.

\section{Proof of the main theorem}

Since the condition $n \geqslant 3$ in Theorem 1.2 is equivalent to the condition $g>3$ for bielliptic curves, we restate the main result in the following form.

THEOREM 3.1. If $g>3$, then the isomorphism class of a bielliptic curve of genus $g$ is determined by the isomorphism class of its Prym variety.

Let $\left(P, \lambda_{P}\right)$ be the Prym variety of dimension $n \geqslant 3$ defined from a bielliptic curve $(C, \sigma)$ of genus $g=n+1$. We will recover the data $\left(E, e_{1}+\cdots+e_{2 n}, \eta\right)$ from the polarized abelian variety $\left(P, \lambda_{P}\right)$, where $E=C / \sigma$ is the quotient curve, $e_{1}+\cdots+e_{2 n}$ is the branch divisor of the covering $\phi: C \rightarrow E$, and $\eta \in \operatorname{Pic}(E)$ is the invertible sheaf with $\phi^{*} \eta \cong \Omega_{C}^{1}$. We remark that $\eta^{\otimes 2} \cong \mathcal{O}_{E}\left(e_{1}+\cdots+e_{2 n}\right)$, and $\eta$ is the invertible sheaf which determines the double covering with branch divisor $e_{1}+\cdots+e_{2 n}$.

Proof of Theorem 3.1. Let $\mathcal{L}$ be an ample invertible sheaf on $P$ which represents the polarization $\lambda_{P}$. We denote the kernel of $\lambda_{P}: P \rightarrow P^{\vee}$ by $K(P)$. By Lemma 2.3, we have $\# K(P)=4$ and $h^{0}(P, \mathcal{L})=2$. We define the subset $\Pi_{\mathcal{L}}$ in the linear pencil $|\mathcal{L}|$ by

$$
\Pi_{\mathcal{L}}=\left\{D \in|\mathcal{L}| \mid t_{x}(D)=D \subset P \text { for some } x \in K(P) \backslash\{0\}\right\},
$$

where $t_{x}$ is the translation by $x \in P$. By Lemma 4.8 , the set $\Pi_{\mathcal{L}}$ has six members for any representative $\mathcal{L}$ of the polarization $\lambda_{P}$. For a member $D \in|\mathcal{L}| \backslash \Pi_{\mathcal{L}}$, we consider the Gauss map

$$
\Psi_{D}: D \backslash D_{\text {sing }} \longrightarrow \mathbf{P}^{n-1}=\operatorname{Grass}\left(n-1, H^{0}\left(P, \Omega_{P}^{1}\right)^{\vee}\right),
$$

where $\Psi_{D}(x)$ is defined by the inclusion $T_{x}(D) \subset T_{x}(P) \cong H^{0}\left(P, \Omega_{P}^{1}\right)^{\vee}$ of the tangent spaces at the point $x \in D \backslash D_{\text {sing }} \subset P$. We set $U_{D}=\operatorname{Bs}|\mathcal{L}| \backslash D_{\text {sing }}$, where Bs $|\mathcal{L}| \subset P$ denotes the set of base points of the pencil $|\mathcal{L}|$. Let $X_{D}^{\prime}=\overline{\Psi_{D}\left(U_{D}\right)} \subset \mathbf{P}^{n-1}$ be the Zariski closure of $\Psi_{D}\left(U_{D}\right) \subset \mathbf{P}^{n-1}$, and let $\nu_{D}: X_{D} \rightarrow X_{D}^{\prime}$ be the normalization. By Lemma 6.1, the variety $U_{D}$ is nonsingular; hence, there is a unique morphism $\psi_{D}: U_{D} \rightarrow X_{D}$ such that $\left.\Psi_{D}\right|_{U_{D}}=\nu_{D} \circ \psi_{D}$. We consider the closed subset $Z_{D}=\overline{\psi_{D}\left(\operatorname{Ram}\left(\psi_{D}\right)\right)} \subset X_{D}$, where $\operatorname{Ram}\left(\psi_{D}\right) \subset U_{D}$ denotes the ramification divisor of $\psi_{D}$. By Proposition 6.2, the divisor $Z_{D}$ has a canonical decomposition $Z_{D}=\bigcup_{i=1}^{2 n} Z_{D, i}$, and there is a unique hyperplane $H_{D, i} \subset \mathbf{P}^{n-1}$ such that $\nu_{D}\left(Z_{D, i}\right) \subset H_{D, i}$ for any $1 \leqslant i \leqslant 2 n$. Then the effective divisor $\nu_{D}^{*} H_{D, i}-Z_{D, i}$ on $X_{D}$ has two irreducible components for general $D \in|\mathcal{L}| \backslash \Pi_{\mathcal{L}}$, and these components coincide for special $D \in|\mathcal{L}| \backslash \Pi_{\mathcal{L}}$. We define the subset $\Pi_{\mathcal{L}}^{\prime}$ in the linear pencil $|\mathcal{L}|$ by

$$
\Pi_{\mathcal{L}}^{\prime}=\left\{D \in|\mathcal{L}| \backslash \Pi_{\mathcal{L}} \mid \nu_{D}^{*} H_{D, i}-Z_{D, i} \text { is irreducible for } 1 \leqslant i \leqslant 2 n\right\} .
$$




\section{PRYM-TORELLI FOR COVERINGS OF ELLIPTIC CURVES}

By Lemma 6.3, the set $\Pi_{\mathcal{L}}^{\prime}$ has four members for any representative $\mathcal{L}$ of the polarization $\lambda_{P}$. For a member $D \in \Pi_{\mathcal{L}}^{\prime}$, we consider the dual variety $\left(X_{D}^{\prime}\right)^{\vee} \subset\left(\mathbf{P}^{n-1}\right)^{\vee}$ of $X_{D}^{\prime} \subset \mathbf{P}^{n-1}$ and the dual variety $H_{D, i}^{\vee} \subset\left(\mathbf{P}^{n-1}\right)^{\vee}$ of $H_{D, i} \subset \mathbf{P}^{n-1}$. By Proposition 6.4 , the variety $H_{D, i}^{\vee}$ is a point on $\left(X_{D}^{\prime}\right)^{\vee}$, and we have an isomorphism

$$
\left(E, e_{1}+\cdots+e_{2 n}, \eta\right) \cong\left(\left(X_{D}^{\prime}\right)^{\vee}, H_{D, 1}^{\vee}+\cdots+H_{D, 2 n}^{\vee},\left.\mathcal{O}_{\left(\mathbf{P}^{n-1}\right) \vee}(1)\right|_{\left(X_{D}^{\prime}\right)^{\vee}}\right) .
$$

\section{Pencil of polarization divisors}

Let $(C, \sigma)$ be a bielliptic curve of genus $g=n+1>3$. For $\delta \in \operatorname{Pic}^{n}(C)$, we define the divisor $W_{\delta} \subset J(C)$ by

$$
W_{\delta}=\left\{L \in \operatorname{Pic}^{0}(C)=J(C) \mid h^{0}(C, L \otimes \delta)>0\right\} .
$$

We remark that the singular locus of $W_{\delta}$ is given by

$$
W_{\delta, \text { sing }}=\left\{L \in \operatorname{Pic}^{0}(C) \mid h^{0}(C, L \otimes \delta)>1\right\},
$$

and $\operatorname{dim} W_{\delta \text {,sing }}=n-3$ (see [AM67, Proposition 8]) because $C$ is not a hyperelliptic curve by Lemma 2.1. Let $\lambda_{C}: J(C) \rightarrow J(C)^{\vee}$ be the homomorphism defined by

$$
\lambda_{C}: J(C) \longrightarrow J(C)^{\vee}=\operatorname{Pic}^{0}(J(C)), \quad x \longmapsto\left[t_{x}^{*} \mathcal{O}_{C}\left(W_{\delta}\right) \otimes \mathcal{O}_{C}\left(-W_{\delta}\right)\right],
$$

which does not depend on the choice of $\delta \in \operatorname{Pic}^{n}(C)$. For $q \in C$, let $\iota_{q}: C \rightarrow J(C)$ be the morphism defined by

$$
\iota_{q}: C \longrightarrow \operatorname{Pic}^{0}(C)=J(C), \quad q^{\prime} \longmapsto\left[\mathcal{O}_{C}\left(q^{\prime}-q\right)\right] .
$$

LEMMA 4.1. We have

$$
x=\iota_{q}^{*}\left[\mathcal{O}_{J(C)}\left(W_{\delta}-W_{\delta+x}\right)\right] \in \operatorname{Pic}^{0}(C)
$$

for any $q \in C$ and $x \in \operatorname{Pic}^{0}(C)$.

Proof. The statement means that $(-1) \circ \lambda_{C}$ is the inverse of the homomorphism $\iota_{q}^{*}: J(C)^{\vee} \rightarrow$ $J(C)$ defined by the pull-back $\iota_{q}^{*}: \operatorname{Pic}^{0}(J(C)) \rightarrow \operatorname{Pic}^{0}(C)$ of invertible sheaves. This is well known [Mil86, Lemma 6.9].

Let $P$ be the kernel of the norm map $N: J(C) \rightarrow J(E)$, and let $D_{\delta} \subset P$ the fiber of the restriction of the norm map $\left.N\right|_{W_{\delta}}: W_{\delta} \rightarrow J(E)$ at $0 \in J(E)$. We denote the restriction of $\mathcal{O}_{J(C)}\left(W_{\delta}\right)$ to $P$ by $\mathcal{L}_{\delta}=\mathcal{O}_{P}\left(D_{\delta}\right)=\left.\mathcal{O}_{J(C)}\left(W_{\delta}\right)\right|_{P}$. Since $W_{\delta}$ is the theta divisor of $J(C)$, the ample invertible sheaf $\mathcal{L}_{\delta}$ represents the polarization $\lambda_{P}$.

Lemma 4.2. The divisor $D_{\delta+\phi^{*} s} \subset P$ is a member of the linear system $\left|\mathcal{L}_{\delta}\right|$ for any $s \in \operatorname{Pic}^{0}(E)$. Proof. By Lemma 4.1, we have

$$
\phi^{*} s=\iota_{q}^{*}\left[\mathcal{O}_{J(C)}\left(W_{\delta}-W_{\delta+\phi^{*} s}\right)\right] \in \operatorname{Pic}^{0}(C)
$$

for $s \in \operatorname{Pic}^{0}(E)$ and $q \in C$. We define $s^{\prime} \in \operatorname{Pic}^{0}(J(E))$ by $s=\iota_{\phi(q)}^{*} s^{\prime}$, where $\iota_{\phi(q)}: E \rightarrow J(E)$ is the isomorphism determined by $\iota_{\phi(q)} \circ \phi=N \circ \iota_{q}$. Then we have

$$
N^{*} s^{\prime}=\left[\mathcal{O}_{J(C)}\left(W_{\delta}-W_{\delta+\phi^{*} s}\right)\right] \in \operatorname{Pic}^{0}(J(C))
$$

because $\phi^{*} s=\iota_{q}^{*} N^{*} s^{\prime}$ and $\iota_{q}^{*}: \operatorname{Pic}^{0}(J(C)) \rightarrow \operatorname{Pic}^{0}(C)$ is an isomorphism. Since $\left.\left(N^{*} s^{\prime}\right)\right|_{P}=0 \in$ $\operatorname{Pic}^{0}(P)$, we have

$$
\mathcal{O}_{P}\left(D_{\delta+\phi^{*} s}\right)=\left.\left.\mathcal{O}_{J(C)}\left(W_{\delta+\phi^{*} s}\right)\right|_{P} \cong \mathcal{O}_{J(C)}\left(W_{\delta}\right)\right|_{P}=\mathcal{L}_{\delta}
$$




\section{A. IKEDA}

We denote the $i$ th symmetric products of $C$ by $C^{(i)}$, and points of $C^{(i)}$ are considered as effective divisors on $C$ of degree $i$. For $\delta \in \operatorname{Pic}^{n}(C)$, we define the morphism $\beta_{\delta}^{i}: C^{(n-2 i)} \times E^{(i)} \rightarrow$ $J(C)$ by

$$
\begin{aligned}
\beta_{\delta}^{i}: C^{(n-2 i)} \times E^{(i)} & \longrightarrow J(C)=\operatorname{Pic}^{0}(C), \\
(\mathbf{q}, \mathbf{p}) & \longmapsto \mathcal{O}_{C}(\mathbf{q}) \otimes \phi^{*} \mathcal{O}_{E}(\mathbf{p}) \otimes \delta^{\vee} .
\end{aligned}
$$

We remark that $W_{\delta}=\operatorname{Image}\left(\beta_{\delta}^{0}\right)$, and we set

$$
B_{\delta}^{i}= \begin{cases}\text { Image }\left(\beta_{\delta}^{i}\right), & 1 \leqslant 2 i \leqslant n, \\ \emptyset, & 2 i>n .\end{cases}
$$

Lemma 4.3. We have $B_{\delta}^{1} \nsubseteq W_{\delta \text {,sing }}$ and $B_{\delta}^{2} \subset W_{\delta \text {,sing }}$.

Proof. Let $B^{1} \subset C^{(n)}$ be the image of the morphism

$$
\beta^{1}: C^{(n-2)} \times E \longrightarrow C^{(n)}, \quad(\mathbf{q}, \phi(q)) \longmapsto \mathbf{q}+q+\sigma(q) .
$$

Since $C$ is not a hyperelliptic curve, we have $\operatorname{dim}\left(\beta_{\delta}^{0}\right)^{-1}\left(W_{\delta \text {,sing }}\right)=n-2<n-1=\operatorname{dim} B^{1}$;

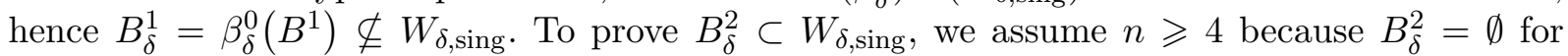
$n=3$. Then it is a consequence of the inequality

$$
\begin{aligned}
h^{0}\left(C, \mathcal{O}_{C}(\mathbf{q}) \otimes \phi^{*} \mathcal{O}_{E}(\mathbf{p})\right) & \geqslant h^{0}\left(C, \phi^{*} \mathcal{O}_{E}(\mathbf{p})\right)=h^{0}\left(E, \phi_{*} \mathcal{O}_{C} \otimes \mathcal{O}_{E}(\mathbf{p})\right) \\
& \geqslant h^{0}\left(E, \mathcal{O}_{E}(\mathbf{p})\right)=2
\end{aligned}
$$

for $(\mathbf{q}, \mathbf{p}) \in C^{(n-4)} \times E^{(2)}$.

Lemma 4.4. For $s \in \operatorname{Pic}^{0}(E)$, we have $D_{\delta}=D_{\delta+\phi^{*} s} \subset P$ if and only if $s=0$ or $s=\eta-N(\delta)$.

Proof. For $L \in P \subset \operatorname{Pic}^{0}(C)$, we have $0=\phi^{*} N(L)=L+\sigma^{*} L \in \operatorname{Pic}^{0}(C)$. Hence we have $D_{\delta}=D_{\delta+\phi^{*}(\eta-N(\delta))}$ because

$$
\begin{aligned}
L \in D_{\delta} & \Longleftrightarrow h^{0}(C, L \otimes \delta)>0 \Longleftrightarrow h^{0}\left(C, \sigma^{*} L \otimes \sigma^{*} \delta\right)>0 \Longleftrightarrow h^{0}\left(C, L^{\vee} \otimes \sigma^{*} \delta\right)>0 \\
& \Longleftrightarrow h^{0}\left(C, \Omega_{C}^{1} \otimes L \otimes \sigma^{*} \delta^{\vee}\right)>0 \Longleftrightarrow L \in D_{\left[\Omega_{C}^{1}\right]-\sigma^{*} \delta}=D_{\delta+\phi^{*}(\eta-N(\delta))} .
\end{aligned}
$$

We assume $D_{\delta}=D_{\delta+\phi^{*} s}$ for $s \neq 0 \in \operatorname{Pic}^{0}(E)$. By [Mum74, §5, Proof of the proposition, p. 338], we have the exact sequence

$$
0 \longrightarrow s \longrightarrow \phi_{*}\left(\phi^{*} s \otimes L \otimes \delta\right) \longrightarrow s \otimes N(L \otimes \delta) \otimes \eta^{\vee} \longrightarrow 0
$$

for $L \in D_{\delta} \backslash B_{\delta}^{1}$. By $h^{0}(E, s)=0$ and $L \in D_{\delta}=D_{\delta+\phi^{*} s}$, we have

$$
h^{0}\left(E, s \otimes N(L \otimes \delta) \otimes \eta^{\vee}\right) \geqslant h^{0}\left(E, \phi_{*}\left(\phi^{*} s \otimes L \otimes \delta\right)\right)>0 .
$$

Since $s \otimes N(L \otimes \delta) \otimes \eta^{\vee}=s \otimes N(\delta) \otimes \eta^{\vee} \in \operatorname{Pic}^{0}(E)$ is effective, we have $s \otimes N(\delta) \otimes \eta^{\vee} \cong \mathcal{O}_{E}$.

Let $B_{\delta} \subset J(C)$ be the subset

$$
B_{\delta}=\bigcap_{s \in \operatorname{Pic}^{0}(E)} W_{\delta+\phi^{*} s} .
$$

Lemma 4.5. We have $B_{\delta} \backslash W_{\delta \text {,sing }}=B_{\delta}^{1} \backslash W_{\delta \text {,sing }}$.

Proof. If $L \in B_{\delta}^{1}$, then $L \otimes \delta \cong \mathcal{O}_{C}(\mathbf{q}+q+\sigma(q))$ for some $\mathbf{q} \in C^{(n-2)}$ and $q \in C$. For $s \in \operatorname{Pic}^{0}(E)$, there is a point $q^{\prime} \in C$ such that $s=\left[\mathcal{O}_{E}\left(\phi\left(q^{\prime}\right)-\phi(q)\right)\right]$. Since $L \otimes \delta \otimes \phi^{*} s \cong \mathcal{O}_{C}\left(\mathbf{q}+q^{\prime}+\sigma\left(q^{\prime}\right)\right)$, we have $h^{0}\left(C, L \otimes \delta \otimes \phi^{*} s\right)>0$ and $L \in W_{\delta+\phi^{*} s}$. Hence the inclusion $B_{\delta}^{1} \subset B_{\delta}$ holds. 


\section{PRYM-TORELLI FOR COVERINGS OF ELLIPTIC CURVES}

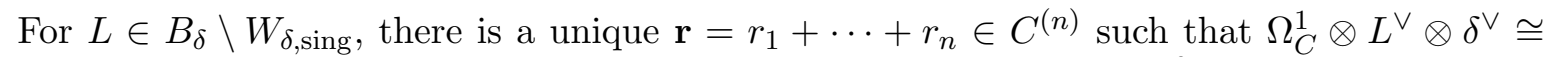
$\mathcal{O}_{C}(\mathbf{r})$ because $h^{0}\left(C, \Omega_{C}^{1} \otimes L^{\vee} \otimes \delta^{\vee}\right)=h^{0}(C, L \otimes \delta)=1$. For $s \in \operatorname{Pic}^{0}(E) \backslash\{0\}$, there is a divisor $\mathbf{q}=q_{1}+\cdots+q_{n} \in C^{(n)}$ such that $L \otimes \delta \otimes \phi^{*} s \cong \mathcal{O}_{C}(\mathbf{q})$ because $L \in B_{\delta} \subset W_{\delta+\phi^{*} s}$. When $s \in \operatorname{Pic}^{0}(E) \backslash\{0\}$ is general, we may assume $\sigma\left(q_{n}\right) \notin\left\{r_{1}, \ldots, r_{n}\right\}$. This implies that $h^{0}\left(C, \Omega_{C}^{1} \otimes L^{\vee} \otimes \delta^{\vee} \otimes \mathcal{O}_{C}\left(-\sigma\left(q_{n}\right)\right)\right)=0$ and $h^{0}\left(C, L \otimes \delta \otimes \mathcal{O}_{C}\left(\sigma\left(q_{n}\right)\right)\right)=1$. Let $\phi\left(q^{\prime}\right) \in E$ be the point determined by $s=\left[\mathcal{O}_{E}\left(\phi\left(q_{n}\right)-\phi\left(q^{\prime}\right)\right)\right]$. Then

$$
L \otimes \delta \otimes \mathcal{O}_{C}\left(\sigma\left(q_{n}\right)\right) \cong \mathcal{O}_{C}\left(q_{1}+\cdots+q_{n-1}+q^{\prime}+\sigma\left(q^{\prime}\right)\right) .
$$

Since $s \neq 0$, we have $\sigma\left(q_{n}\right) \in\left\{q_{1}, \ldots, q_{n-1}\right\}$ and $L \in B_{\delta}^{1}$.

LEMMA 4.6. The map

$$
\operatorname{Pic}^{0}(E) \longrightarrow\left|\mathcal{L}_{\delta}\right|, \quad s \longmapsto D_{\delta+\phi^{*} s}
$$

is a double covering, and the base locus $\operatorname{Bs}\left|\mathcal{L}_{\delta}\right|$ of the linear system $\left|\mathcal{L}_{\delta}\right|$ is $B_{\delta} \cap P$, which is of dimension $n-2$.

Proof. The map is well defined by Lemma 4.2. Since $\operatorname{dim}\left|\mathcal{L}_{\delta}\right|=1$, it is a double covering by Lemma 4.4. Hence we have

$$
\mathrm{Bs}\left|\mathcal{L}_{\delta}\right|=\bigcap_{s \in \operatorname{Pic}^{0}(E)} D_{\delta+\phi^{*} s}=B_{\delta} \cap P .
$$

By Lemma 4.3, the variety $B_{\delta}^{1}$ is irreducible of dimension $n-1$. Since the restriction of the norm map $\left.N\right|_{B_{\delta}^{1}}: B_{\delta}^{1} \rightarrow J(E)$ is surjective, we have $\operatorname{dim} B_{\delta}^{1} \cap P=n-2$, hence $\operatorname{dim} B_{\delta} \cap P=n-2$ by Lemma 4.5 .

LEMma 4.7. Let $\mathcal{L}$ be an ample invertible sheaf which represents the polarization $\lambda_{P}$ on $P$; then there is a $\delta \in \operatorname{Pic}^{n}(C)$ such that $N(\delta)=\eta$ and $\mathcal{L} \cong \mathcal{L}_{\delta}$.

Proof. For any $\delta^{\prime} \in \operatorname{Pic}^{n}(C)$, we have $\mathcal{L} \otimes \mathcal{L}_{\delta^{\prime}}^{\vee} \in \operatorname{Pic}^{0}(P)$ because $\mathcal{L}_{\delta^{\prime}}$ gives the same polarization as $\lambda_{P}$. Then $\mathcal{L} \cong t_{x}^{*} \mathcal{L}_{\delta^{\prime}} \cong \mathcal{L}_{\delta^{\prime}+x}$ for some $x \in P$. Let $s \in \operatorname{Pic}^{0}(E)$ be a point with $2 s=$ $\eta-N\left(\delta^{\prime}+x\right)$. For $\delta=\delta^{\prime}+x+\phi^{*} s$, we have $N(\delta)=\eta$ and $\mathcal{L} \cong \mathcal{L}_{\delta}$.

For an ample invertible sheaf $\mathcal{L}$ which represents the polarization $\lambda_{P}$, we define a subset in the linear system $|\mathcal{L}|$ by

$$
\Pi_{\mathcal{L}}=\left\{D \in|\mathcal{L}| \mid t_{x}(D)=D \text { for some } x \in K(P) \backslash\{0\}\right\},
$$

where $K(P)$ is the kernel of the polarization $\lambda_{P}$.

LEMma 4.8. We have $\# \Pi_{\mathcal{L}}=6$.

Proof. By Lemma 4.7, there is a $\delta \in \operatorname{Pic}^{n}(C)$ such that $N(\delta)=\eta$ and $\mathcal{L} \cong \mathcal{L}_{\delta}$. For any $D \in\left|\mathcal{L}_{\delta}\right|$, by Lemma 4.6, there is an $s \in \mathrm{Pic}^{0}(E)$ such that $D=D_{\delta+\phi^{*} s}$. If $D_{\delta+\phi^{*} s} \in \Pi_{\mathcal{L}_{\delta}}$, then by Lemma 2.3, there is a $t \in J(E)_{2} \backslash\{0\}$ such that $t_{\phi^{*} t}\left(D_{\delta+\phi^{*} s}\right)=D_{\delta+\phi^{*} s}$. Since $t_{\phi^{*} t}\left(D_{\delta+\phi^{*} s}\right)=$ $D_{\delta+\phi^{*} s+\phi^{*}(-t)}$ and $t \neq 0$, by Lemma 4.4, we have $-t=\eta-N\left(\delta+\phi^{*} s\right)$, hence $t=2 s$. This means that

$$
\Pi_{\mathcal{L}_{\delta}}=\left\{D_{\delta+\phi^{*} s} \in\left|\mathcal{L}_{\delta}\right| \mid s \in J(E)_{4} \backslash J(E)_{2}\right\} .
$$

Since $\#\left(J(E)_{4} \backslash J(E)_{2}\right)=12$ and $D_{\delta+\phi^{*} s}=D_{\delta-\phi^{*} s}$, we have $\# \Pi_{\mathcal{L}}=\# \Pi_{\mathcal{L}_{\delta}}=6$. 


\section{A. IKEDA}

\section{Gauss maps}

\subsection{Gauss maps for the Jacobian and for the Prym variety}

Let

$$
\Psi_{J(C), \delta}: W_{\delta} \backslash W_{\delta, \text { sing }} \longrightarrow \mathbf{P}\left(H^{0}\left(C, \Omega_{C}^{1}\right)^{\vee}\right)=\operatorname{Grass}\left(n, H^{0}\left(C, \Omega_{C}^{1}\right)^{\vee}\right)
$$

be the Gauss map for the subvariety $W_{\delta} \subset J(C)$. We note that the projective space $\mathbf{P}(V)$ is the set of subspaces of codimension 1 in the vector space $V$. For $L \in W_{\delta} \backslash W_{\delta \text {,sing }}$, the tangent space $T_{L}\left(W_{\delta}\right)$ of $W_{\delta}$ at $L$ defines the image $\Psi_{J(C), \delta}(L)$ by the natural identifications

$$
T_{L}\left(W_{\delta}\right) \subset T_{L}(J(C)) \cong\left(\Omega_{J(C)}^{1} \mid L\right)^{\vee} \cong H^{0}\left(J(C), \Omega_{J(C)}^{1}\right)^{\vee} \cong H^{0}\left(C, \Omega_{C}^{1}\right)^{\vee} .
$$

Lemma 5.1. For $L \in W_{\delta} \backslash W_{\delta \text {,sing }}$, the image $\Psi_{J(C), \delta}(L)$ of the Gauss map is identified with the canonical divisor

$$
\mathbf{q}+\mathbf{q}^{\prime} \in\left|\Omega_{C}^{1}\right|=\operatorname{Grass}\left(1, H^{0}\left(C, \Omega_{C}^{1}\right)\right) \cong \mathbf{P}\left(H^{0}\left(C, \Omega_{C}^{1}\right)^{\vee}\right),
$$

where $\mathbf{q} \in C^{(n)}$ and $\mathbf{q}^{\prime} \in C^{(n)}$ are uniquely determined by $L \otimes \delta \cong \mathcal{O}_{C}(\mathbf{q})$ and $\Omega_{C}^{1} \otimes L^{\vee} \otimes \delta^{\vee} \cong$ $\mathcal{O}_{C}\left(\mathbf{q}^{\prime}\right)$.

Proof. This is a special case of Proposition 4.2 in [ACGH85, Chapter IV].

Lemma 5.2. Let $K \in\left|\Omega_{C}^{1}\right|$ be an effective canonical divisor. If $q_{1}+\sigma\left(q_{1}\right) \leqslant K$ for some $q_{1} \in C$, then $K=\sum_{i=1}^{n}\left(q_{i}+\sigma\left(q_{i}\right)\right)$ for some $q_{2}, \ldots, q_{n} \in C$.

Proof. We assume

$$
K=\sum_{i=1}^{m}\left(q_{i}+\sigma\left(q_{i}\right)\right)+\sum_{j=1}^{2 n-2 m} r_{j}
$$

and $\sigma\left(r_{i}\right) \neq r_{j}$ for $i \neq j$. By [Mum74, $\S 5$, Proof of the proposition, p. 338], we have the exact sequence

$$
0 \longrightarrow A \longrightarrow \phi_{*}\left(\phi^{*} A \otimes L\right) \longrightarrow A \otimes N(L) \otimes \eta^{\vee} \longrightarrow 0
$$

for $A=\mathcal{O}_{E}\left(\sum_{i=1}^{m} \phi\left(q_{i}\right)\right)$ and $L=\mathcal{O}_{C}\left(\sum_{j=1}^{2 n-2 m} r_{j}\right)$. By the assumption $m \geqslant 1$, we have $h^{0}(E, A)=m$, hence

$$
\begin{aligned}
h^{0}\left(E, A \otimes N(L) \otimes \eta^{\vee}\right) & \geqslant h^{0}\left(E, \phi_{*}\left(\phi^{*} A \otimes L\right)\right)-h^{0}(E, A)=n+1-m \\
& =\operatorname{deg}\left(A \otimes N(L) \otimes \eta^{\vee}\right)+1 .
\end{aligned}
$$

This implies that $m=n$ and $A \cong \eta$.

By the injective homomorphism

$$
H^{0}(E, \eta) \longrightarrow H^{0}\left(C, \phi^{*} \eta\right) \cong H^{0}\left(C, \Omega_{C}^{1}\right)
$$

we have the closed immersion

$$
\iota: \mathbf{P}\left(H^{0}(E, \eta)^{\vee}\right) \longrightarrow \mathbf{P}\left(H^{0}\left(C, \Omega_{C}^{1}\right)^{\vee}\right) .
$$

Lemma 5.3. For $L \in B_{\delta} \backslash W_{\delta \text {,sing, we have }}$

$$
\Psi_{J(C), \delta}(L) \in \iota\left(\mathbf{P}\left(H^{0}(E, \eta)^{\vee}\right)\right) \subset \mathbf{P}\left(H^{0}\left(C, \Omega_{C}^{1}\right)^{\vee}\right) .
$$

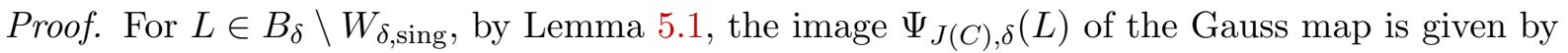

$$
\mathbf{q}+\mathbf{q}^{\prime} \in\left|\Omega_{C}^{1}\right| \cong \mathbf{P}\left(H^{0}\left(C, \Omega_{C}^{1}\right)^{\vee}\right),
$$




\section{PRYM-TORELLI FOR COVERINGS OF ELLIPTIC CURVES}

where $\mathbf{q}=q_{1}+\cdots+q_{n} \in C^{(n)}$ and $\mathbf{q}^{\prime} \in C^{(n)}$ are uniquely determined by $L \otimes \delta \cong \mathcal{O}_{C}(\mathbf{q})$ and $\Omega_{C}^{1} \otimes L^{\vee} \otimes \delta^{\vee} \cong \mathcal{O}_{C}\left(\mathbf{q}^{\prime}\right)$. Since $L \in B_{\delta}$, by Lemma 4.5, we have $\sigma\left(q_{i}\right)=q_{j}$ for some $i \neq j$. By Lemma 5.2, we have $\Psi_{J(C), \delta}(L) \in \iota\left(\mathbf{P}\left(H^{0}(E, \eta)^{\vee}\right)\right)$.

Let

$$
\Psi_{P, \delta}: D_{\delta} \backslash D_{\delta, \text { sing }} \rightarrow \mathbf{P}\left(H^{0}\left(P, \Omega_{P}^{1}\right)^{\vee}\right)=\operatorname{Grass}\left(n-1, H^{0}\left(P, \Omega_{P}^{1}\right)^{\vee}\right)
$$

be the Gauss map for the subvariety $D_{\delta} \subset P$. For $L \in D_{\delta} \backslash D_{\delta \text {,sing }}$, the tangent space $T_{L}\left(D_{\delta}\right)$ of $D_{\delta}$ at $L$ defines the image $\Psi_{P, \delta}(L)$ by the natural identifications

$$
T_{L}\left(D_{\delta}\right) \subset T_{L}(P) \cong\left(\left.\Omega_{P}^{1}\right|_{L}\right)^{\vee} \cong H^{0}\left(P, \Omega_{P}^{1}\right)^{\vee} .
$$

For $L \in P$, the tangent space $T_{L}(P)$ of $P$ at $L$ is naturally identified with the orthogonal complement

$$
V_{P}=\left(\phi^{*} H^{0}\left(E, \Omega_{E}^{1}\right)\right)^{\perp} \subset H^{0}\left(C, \Omega_{C}^{1}\right)^{\vee} \cong T_{L}(J(C))
$$

of $\phi^{*} H^{0}\left(E, \Omega_{E}^{1}\right) \subset H^{0}\left(C, \Omega_{C}^{1}\right)$, and it corresponds to the ramification divisor $\operatorname{Ram}(\phi) \in\left|\Omega_{C}^{1}\right|$ of the covering $\phi: C \rightarrow E$. We define the finite subset $\Sigma_{\delta} \subset J(C)$ by

$$
\Sigma_{\delta}=\left\{\beta_{\delta}^{0}(\mathbf{r}) \in J(C) \mid \mathbf{r} \leqslant \operatorname{Ram}(\phi), \mathbf{r} \in C^{(n)}\right\} .
$$

Lemma 5.4. We have $D_{\delta \text {,sing }}=\left(W_{\delta \text {,sing }} \cup \Sigma_{\delta}\right) \cap D_{\delta}$.

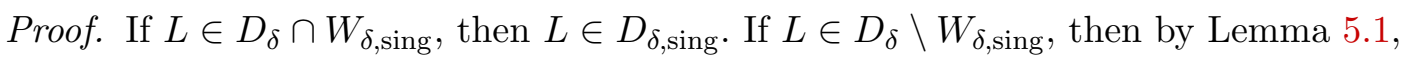

$$
L \in D_{\delta, \text { sing }} \Longleftrightarrow T_{L}\left(W_{\delta}\right)=T_{L}(P) \subset T_{L}(J(C)) \Longleftrightarrow L \in \Sigma_{\delta} .
$$

Lemma 5.5. We have $\left(B_{\delta} \cap P\right) \backslash W_{\delta \text {,sing }}=\left(B_{\delta} \cap P\right) \backslash D_{\delta \text {,sing. }}$.

Proof. By Lemma 4.5, we have $\left(B_{\delta} \backslash W_{\delta \text {,sing }}\right) \cap \Sigma_{\delta}=\left(B_{\delta}^{1} \backslash W_{\delta \text {,sing }}\right) \cap \Sigma_{\delta}$, and it is empty because $\operatorname{Ram}(\phi)$ is reduced. Hence by Lemma 5.4, we have

$$
W_{\delta, \text { sing }} \cap B_{\delta} \cap P=W_{\delta, \text { sing }} \cap B_{\delta} \cap D_{\delta}=D_{\delta, \text { sing }} \cap B_{\delta}=D_{\delta, \text { sing }} \cap B_{\delta} \cap P .
$$

We denote by

$$
\begin{aligned}
\pi: \mathbf{P}\left(H^{0}\left(C, \Omega_{C}^{1}\right)^{\vee}\right) \backslash\left\{V_{P}\right\} & \longrightarrow \mathbf{P}\left(H^{0}\left(P, \Omega_{P}^{1}\right)^{\vee}\right) \\
{\left[V \subset H^{0}\left(C, \Omega_{C}^{1}\right)^{\vee}\right] } & \longmapsto\left[V \cap V_{P} \subset V_{P} \cong H^{0}\left(P, \Omega_{P}^{1}\right)^{\vee}\right]
\end{aligned}
$$

the projection, where $V_{P}=\left(\phi^{*} H^{0}\left(E, \Omega_{E}^{1}\right)\right)^{\perp} \subset H^{0}\left(C, \Omega_{C}^{1}\right)^{\vee}$ is the image of the dual of the restriction

$$
H^{0}\left(C, \Omega_{C}^{1}\right) \cong H^{0}\left(J(C), \Omega_{J(C)}^{1}\right) \rightarrow H^{0}\left(P, \Omega_{P}^{1}\right) .
$$

Lemma 5.6. We have $\Psi_{P, \delta}(L)=\pi \circ \Psi_{J(C), \delta}(L)$ for $L \in D_{\delta} \backslash D_{\delta \text {,sing. }}$.

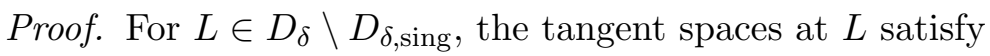

$$
T_{L}\left(W_{\delta}\right) \cap T_{L}(P)=T_{L}\left(D_{\delta}\right) \subset T_{L}(J(C))
$$

because $W_{\delta} \cap P=D_{\delta} \subset J(C)$. Since $T_{L}(P) \subset T_{L}(J(C))$ is identified with $V_{P} \subset H^{0}\left(C, \Omega_{C}^{1}\right)^{\vee}$ by $T_{L}(J(C)) \cong H^{0}\left(C, \Omega_{C}^{1}\right)^{\vee}$, we have $\Psi_{P, \delta}(L)=\pi \circ \Psi_{J(C), \delta}(L)$.

By Lemma 5.3, we have the morphism

$$
\Psi_{J(C), \delta}^{B}: B_{\delta} \backslash W_{\delta, \text { sing }} \longrightarrow \mathbf{P}\left(H^{0}(E, \eta)^{\vee}\right)
$$

satisfying $\iota \circ \Psi_{J(C), \delta}^{B}=\Psi_{J(C), \delta}$. 


\section{A. IKEDA}

Lemma 5.7. The restriction $\left.\Psi_{P, \delta}\right|_{\left(B_{\delta} \cap P\right) \backslash D_{\delta \text {,sing }}}$ of the Gauss map $\Psi_{P, \delta}$ is identified with the restriction $\left.\Psi_{J(C), \delta}^{B}\right|_{\left(B_{\delta} \cap P\right) \backslash D_{\delta, \text { sing }}}$ of $\Psi_{J(C), \delta}^{B}$ by the isomorphism

$$
\pi \circ \iota: \mathbf{P}\left(H^{0}(E, \eta)^{\vee}\right) \stackrel{\cong}{\longrightarrow} \mathbf{P}\left(H^{0}\left(P, \Omega_{P}^{1}\right)^{\vee}\right) \text {. }
$$

Proof. Since the composition

$$
H^{0}(E, \eta) \hookrightarrow H^{0}\left(C, \Omega_{C}^{1}\right) \cong H^{0}\left(J(C), \Omega_{J(C)}^{1}\right) \rightarrow H^{0}\left(P, \Omega_{P}^{1}\right)
$$

is an isomorphism, the result is a consequence of Lemma 5.6.

\subsection{Description for the restricted Gauss maps}

Let $\gamma_{\delta}: E^{(n-2)} \times E \rightarrow J(E)$ be the morphism defined by

$$
\begin{aligned}
\gamma_{\delta}: E^{(n-2)} \times E & \longrightarrow \operatorname{Pic}^{0}(E)=J(E), \\
(\mathbf{p}, p) & \longmapsto \mathcal{O}_{E}(\mathbf{p}+2 p) \otimes(N(\delta))^{\vee} .
\end{aligned}
$$

Let $X_{\delta} \subset E^{(n-2)} \times E$ be the fiber of $\gamma_{\delta}$ at $0 \in J(E)$, and let $Y_{\delta} \subset C^{(n-2)} \times E$ be the fiber of the composition

$$
C^{(n-2)} \times E \stackrel{\phi^{(n-2)} \times \operatorname{id}_{E}}{\longrightarrow} E^{(n-2)} \times E \stackrel{\gamma_{\delta}}{\longrightarrow} J(E)
$$

at $0 \in J(E)$. We denote by $\psi_{\delta}: Y_{\delta} \rightarrow X_{\delta}$ the morphism induced by $\phi^{(n-2)} \times \operatorname{id}_{E}$. Let $\nu_{\delta}: X_{\delta} \rightarrow$ $|\eta| \cong \mathbf{P}\left(H^{0}(E, \eta)^{\vee}\right)$ be the morphism defined by

$$
\nu_{\delta}: X_{\delta} \longrightarrow|\eta|, \quad(\mathbf{p}, p) \longmapsto \mathbf{p}+p+t_{\eta-N(\delta)}(p),
$$

where $t_{\eta-N(\delta)}(p) \in E$ is the point determined by

$$
\left[\mathcal{O}_{E}\left(t_{\eta-N(\delta)}(p)\right)\right]=\left[\mathcal{O}_{E}(p)\right]+\eta-N(\delta) \in \operatorname{Pic}(E) .
$$

We remark that $\beta_{\delta}^{1}\left(Y_{\delta}\right)=B_{\delta}^{1} \cap P \subset J(C)$, and we set

$$
Y_{\delta}^{\circ}=\left(\beta_{\delta}^{1}\right)^{-1}\left(\left(B_{\delta}^{1} \cap P\right) \backslash D_{\delta, \text { sing }}\right)=\left(\beta_{\delta}^{1}\right)^{-1}\left(\left(B_{\delta} \cap P\right) \backslash D_{\delta \text {,sing }}\right) .
$$

LEMma 5.8. The diagram

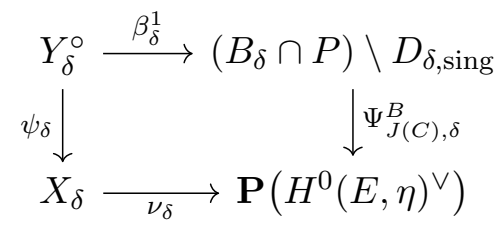

is commutative.

Proof. Let $L \in \operatorname{Pic}^{0}(C)$ be the invertible sheaf which represents the point $\beta_{\delta}^{1}(y) \in J(C)$ for $y=(\mathbf{q}, \phi(q)) \in Y_{\delta}^{\circ}$. Then $\mathbf{q}+q+\sigma(q) \in C^{(n)}$ is the unique effective divisor with $L \cong \mathcal{O}_{C}(\mathbf{q}+$ $q+\sigma(q)) \otimes \delta^{\vee}$. Since

$$
\nu_{\delta} \circ \psi_{\delta}(y)=\phi^{(n-2)}(\mathbf{q})+\phi(q)+t_{\eta-N(\delta)}(\phi(q)) \in|\eta|,
$$

we have

$$
\mathbf{q}+\sigma(\mathbf{q})+q+\sigma(q)+r+\sigma(r) \in\left|\Omega_{C}^{1}\right|,
$$

where $r \in C$ is given by $\phi(r)=t_{\eta-N(\delta)}(\phi(q))$. Then $\sigma(\mathbf{q})+r+\sigma(r) \in C^{(n)}$ is the unique effective divisor with $\mathcal{O}_{C}(\sigma(\mathbf{q})+r+\sigma(r)) \cong \Omega_{C}^{1} \otimes L^{\vee} \otimes \delta^{\vee}$, and by Lemma 5.1, the image $\Psi_{J(C), \delta}^{B}(L)$ is equal to $\nu_{\delta} \circ \psi_{\delta}(y)$. 


\section{PRYM-TORELLI FOR COVERINGS OF ELLIPTIC CURVES}

Lemma 5.9. The projective varieties $X_{\delta}$ and $Y_{\delta}$ are nonsingular.

Proof. We fix a point $p_{0} \in E$. Let $\gamma_{\delta, p_{0}}: E^{(n-2)} \rightarrow J(E)$ be the morphism defined by

$$
\begin{aligned}
\gamma_{\delta, p_{0}}: E^{(n-2)} & \longrightarrow \operatorname{Pic}^{0}(E)=J(E), \\
\mathbf{p} & \longmapsto \mathcal{O}_{E}\left(\mathbf{p}+2 p_{0}\right) \otimes(N(\delta))^{\vee} .
\end{aligned}
$$

Then $\psi_{\delta}: Y_{\delta} \rightarrow X_{\delta}$ is the base change of $\phi^{(n-2)}: C^{(n-2)} \rightarrow E^{(n-2)}$ by the étale covering of degree 4:

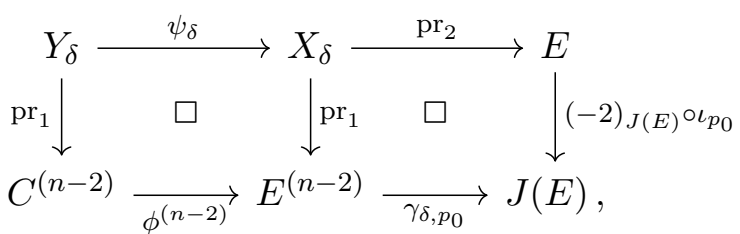

where $(-2)_{J(E)} \circ \iota_{p_{0}}: E \rightarrow J(E)$ is given by

$$
(-2)_{J(E)} \circ \iota_{p_{0}}: E \longrightarrow \operatorname{Pic}^{0}(E)=J(E), \quad p \longmapsto \mathcal{O}_{E}\left(2 p_{0}-2 p\right) \text {. }
$$

LEMma 5.10. The map $\left.\beta_{\delta}^{1}\right|_{Y_{\delta}^{\circ}}: Y_{\delta}^{\circ} \rightarrow\left(B_{\delta} \cap P\right) \backslash D_{\delta \text {,sing }}$ is an isomorphism. In particular, $\left(B_{\delta} \cap\right.$ $P) \backslash D_{\delta \text {,sing }}$ is a nonsingular variety.

Proof. By Lemma 5.5, the image $\beta_{\delta}^{1}\left(Y_{\delta}^{\circ}\right)=\left(B_{\delta} \cap P\right) \backslash D_{\delta \text {,sing }}$ is a closed subset in $W_{\delta} \backslash W_{\delta \text {,sing. }}$. We show that $\left.\beta_{\delta}^{1}\right|_{Y^{\circ}}: Y_{\delta}^{\circ} \rightarrow W_{\delta} \backslash W_{\delta \text {,sing }}$ is a closed immersion. Let $\beta^{1}: C^{(n-2)} \times E \rightarrow C^{(n)}$ be the morphism given in the proof of Lemma 4.3. Since $\beta_{\delta}^{1}=\beta_{\delta}^{0} \circ \beta^{1}$ and $\beta_{\delta}^{0}: C^{(n)} \rightarrow J(C)$ induces the isomorphism

$$
\beta_{\delta}^{0}: C^{(n)} \backslash\left(\beta_{\delta}^{0}\right)^{-1}\left(W_{\delta, \text { sing }}\right) \stackrel{\cong}{\longrightarrow} W_{\delta} \backslash W_{\delta \text {,sing }},
$$

it is enough to show that the finite morphism

$$
\beta^{1}:\left(C^{(n-2)} \times E\right) \backslash\left(\beta_{\delta}^{1}\right)^{-1}\left(W_{\delta, \text { sing }}\right) \longrightarrow C^{(n)} \backslash\left(\beta_{\delta}^{0}\right)^{-1}\left(W_{\delta, \text { sing }}\right)
$$

is a closed immersion. We remark that it is injective by Lemma 4.3. For $y=\left(\mathbf{q}, \phi\left(q_{0}\right)\right) \in$ $\left(C^{(n-2)} \times E\right) \backslash\left(\beta_{\delta}^{1}\right)^{-1}\left(W_{\delta \text {,sing }}\right)$, we prove that the homomorphism

$$
d \beta^{1}: T_{y}\left(C^{(n-2)} \times E\right) \longrightarrow T_{\beta^{1}(y)}\left(C^{(n)}\right)
$$

on the tangent spaces is injective. If $y=\left(\mathbf{q}, \phi\left(q_{0}\right)\right)$ does not satisfy $\phi\left(q_{0}\right) \leqslant \phi(\mathbf{q})$, then the point $y^{\prime}=\left(\mathbf{q}, q_{0}+\sigma\left(q_{0}\right)\right) \in C^{(n-2)} \times C^{(2)}$ is not contained in the ramification divisor of the natural covering $C^{(n-2)} \times C^{(2)} \rightarrow C^{(n)}$. Since the morphism

$$
E \longrightarrow C^{(2)}, \quad \phi(q) \longmapsto q+\sigma(q)
$$

is a closed immersion, the homomorphism

$$
T_{y}\left(C^{(n-2)} \times E\right) \longleftrightarrow T_{y^{\prime}}\left(C^{(n-2)} \times C^{(2)}\right) \cong T_{\beta^{1}(y)}\left(C^{(n)}\right)
$$

is injective. When $y=\left(\mathbf{q}, \phi\left(q_{0}\right)\right)$ satisfies $\phi\left(q_{0}\right) \leqslant \phi(\mathbf{q})$, we can prove the injectivity of $d \beta^{1}$ in a similar way, by using the fact that the morphism

$$
C \times E \longrightarrow C^{(3)}, \quad\left(q^{\prime}, \phi(q)\right) \longmapsto q^{\prime}+q+\sigma(q)
$$

is a closed immersion.

Let $X_{\delta}^{\prime}=\overline{\Psi_{J(C), \delta}^{B}\left(\left(B_{\delta} \cap P\right) \backslash D_{\delta \text {,sing }}\right)}$ be the Zariski closure of the image of the restricted Gauss map $\left.\Psi_{J(C), \delta}^{B}\right|_{\left(B_{\delta} \cap P\right) \backslash D_{\delta, \text { sing }}}$ in $\mathbf{P}\left(H^{0}(E, \eta)^{\vee}\right)$. 


\section{A. IKEDA}

LEMMA 5.11. We have $\nu_{\delta}\left(X_{\delta}\right)=X_{\delta}^{\prime}$.

Proof. By Lemma 5.8, we have

$$
\Psi_{J(C), \delta}^{B}\left(\left(B_{\delta} \cap P\right) \backslash D_{\delta \text {,sing }}\right)=\nu_{\delta}\left(\psi_{\delta}\left(Y_{\delta}^{\circ}\right)\right) \subset \nu_{\delta}\left(X_{\delta}\right),
$$

hence $X_{\delta}^{\prime}=\overline{\nu_{\delta}\left(\psi_{\delta}\left(Y_{\delta}^{\circ}\right)\right)} \subset \nu_{\delta}\left(X_{\delta}\right)$ and $Y_{\delta}^{\circ} \subset\left(\nu_{\delta} \circ \psi_{\delta}\right)^{-1}\left(X_{\delta}^{\prime}\right)$. Since $Y_{\delta}^{\circ}$ is dense in $Y_{\delta}$, we have $Y_{\delta} \subset\left(\nu_{\delta} \circ \psi_{\delta}\right)^{-1}\left(X_{\delta}^{\prime}\right)$ and $\nu_{\delta}\left(X_{\delta}\right)=\left(\nu_{\delta} \circ \psi_{\delta}\right)\left(Y_{\delta}\right) \subset X_{\delta}^{\prime}$.

Lemma 5.12. If $N(\delta)-\eta \notin J(E)_{2} \backslash\{0\}$, then $\nu_{\delta}: X_{\delta} \rightarrow X_{\delta}^{\prime}$ is the normalization of $X_{\delta}^{\prime}$.

Proof. Let $U \subset E^{(n)}$ be the dense open subset whose complement $E^{(n)} \backslash U$ is the set of the point $\mathbf{p} \in E^{(n)}$ satisfying the condition

$$
\left\{\begin{array}{l}
\mathbf{p} \geqslant 2 p+t_{\eta-N(\delta)}(p), \\
\mathbf{p} \geqslant 2 p+t_{N(\delta)-\eta}(p), \\
\mathbf{p} \geqslant p+t_{\eta-N(\delta)}(p)+t_{N(\delta)-\eta}(p), \\
\text { or } \mathbf{p} \geqslant p+t_{\eta-N(\delta)}(p)+p^{\prime}+t_{\eta-N(\delta)}\left(p^{\prime}\right)
\end{array} \quad \text { for some } p, p^{\prime} \in E .\right.
$$

By the natural inclusion $X_{\delta}^{\prime} \subset|\eta| \subset E^{(n)}$, the subset $X_{\delta}^{\prime} \cap U$ is dense open in $X_{\delta}^{\prime}$. We show that the morphism $\nu_{\delta}: \nu_{\delta}^{-1}(U) \rightarrow U$ is a closed immersion. For $\mathbf{p} \in X_{\delta}^{\prime} \cap U$, we assume

$$
\mathbf{p} \geqslant p+t_{\eta-N(\delta)}(p) \text { and } \mathbf{p} \geqslant p^{\prime}+t_{\eta-N(\delta)}\left(p^{\prime}\right)
$$

for some $p \neq p^{\prime} \in E$. Since $\mathbf{p} \in U$, we have

$$
t_{\eta-N(\delta)}(p)=p^{\prime} \quad \text { and } \quad p=t_{\eta-N(\delta)}\left(p^{\prime}\right),
$$

hence $N(\delta)-\eta \in J(E)_{2} \backslash\{0\}$. This means that $\nu_{\delta}: \nu_{\delta}^{-1}(U) \rightarrow X_{\delta}^{\prime} \cap U$ is bijective if $N(\delta)-\eta \notin$ $J(E)_{2} \backslash\{0\}$. In the following, we prove that the homomorphism

$$
d \nu_{\delta}: T_{x}\left(X_{\delta}\right) \longrightarrow T_{\nu_{\delta}(x)}\left(E^{(n)}\right)
$$

on the tangent spaces is injective for $x \in \nu_{\delta}^{-1}(U)$. Let $\tilde{\nu}_{\delta}: E^{(n-2)} \times E \rightarrow E^{(n-2)} \times E^{(2)}$ be the morphism defined by

$$
\begin{aligned}
\tilde{\nu}_{\delta}: E^{(n-2)} \times E & \longrightarrow E^{(n-2)} \times E^{(2)}, \\
(\mathbf{p}, p) & \longmapsto\left(\mathbf{p}, p+t_{\eta-N(\delta)}(p)\right) .
\end{aligned}
$$

If $N(\delta)-\eta \notin J(E)_{2} \backslash\{0\}$, then the morphism $\tilde{\nu}_{\delta}$ is a closed immersion. For $x \in \nu_{\delta}^{-1}(U)$, the image $\tilde{\nu}_{\delta}(x)$ is not contained in the ramification divisor of the natural covering $E^{(n-2)} \times E^{(2)} \rightarrow E^{(n)}$. Hence the homomorphism

$$
T_{x}\left(X_{\delta}\right) \subset T_{x}\left(E^{(n-2)} \times E\right) \longleftrightarrow T_{\tilde{\nu}_{\delta}(x)}\left(E^{(n)} \times E^{(2)}\right) \cong T_{\nu_{\delta}(x)}\left(E^{(n)}\right)
$$

is injective. By Lemma 5.9, the finite birational morphism $\nu_{\delta}: X_{\delta} \rightarrow X_{\delta}^{\prime}$ gives the normalization of $X_{\delta}^{\prime}$.

Remark 5.13. If $N(\delta)-\eta \in J(E)_{2} \backslash\{0\}$, then $\nu_{\delta}: X_{\delta} \rightarrow X_{\delta}^{\prime}$ is a covering of degree 2 .

\subsection{The branch locus of the restricted Gauss maps}

Let $R_{\delta} \subset Y_{\delta}$ be the divisor defined by

$$
R_{\delta}=\left\{\left(q_{1}+\cdots+q_{n-2}, p\right) \in Y_{\delta} \mid q_{i}=\sigma\left(q_{j}\right) \text { for some } i \neq j\right\} .
$$

LEMMA 5.14. We have $\beta_{\delta}^{1}\left(R_{\delta}\right) \subset W_{\delta \text {,sing }}$. 


\section{PRYM-TORELLI FOR COVERINGS OF ELLIPTIC CURVES}

Proof. This is a consequence of Lemma 4.3 because $\beta_{\delta}^{1}\left(R_{\delta}\right) \subset B_{\delta}^{2}$.

Let $S_{\delta, r} \subset Y_{\delta}$ be the divisor defined by

$$
S_{\delta, r}=\left\{(\mathbf{q}, p) \in Y_{\delta} \mid \mathbf{q} \geqslant r\right\}
$$

for $r \in \operatorname{Ram}(\phi)$. Then the ramification divisor of $\psi_{\delta}: Y_{\delta} \rightarrow X_{\delta}$ is

$$
\operatorname{Ram}\left(\psi_{\delta}\right)=R_{\delta} \cup \bigcup_{r \in \operatorname{Ram}(\phi)} S_{\delta, r} .
$$

Lemma 5.15. We have $\beta_{\delta}^{1}\left(S_{\delta, r}\right) \nsubseteq W_{\delta \text {,sing }}$ and, moreover, $\beta_{\delta}^{1}\left(S_{\delta, r}\right) \cap W_{\delta \text {,sing }}=\emptyset$ if $n=3$.

Proof. Let $W_{\delta, r}^{1} \subset J(C)$ be the subvariety defined by

$$
W_{\delta, r}^{1}=\left\{L \in \operatorname{Pic}^{0}(C) \mid h^{0}\left(C, \mathcal{L} \otimes \mathcal{O}_{C}(-r) \otimes \delta\right)>1\right\},
$$

and let $T_{\delta, r} \subset J(C)$ be the image of the morphism

$$
\begin{aligned}
C^{(n-3)} \times E & \longrightarrow \operatorname{Pic}^{0}(C)=J(C), \\
(\mathbf{q}, \phi(q)) & \longmapsto \mathcal{O}_{C}(\mathbf{q}+r+q+\sigma(q)) \otimes \delta^{\vee} .
\end{aligned}
$$

Since $C$ is not a hyperelliptic curve, by Martens's theorem [Mar67, Theorem 1], we have $\operatorname{dim} W_{\delta, r}^{1} \leqslant$ $n-4$ and $T_{\delta, r} \nsubseteq W_{\delta, r}^{1}$, hence $\operatorname{dim} T_{\delta, r}=n-2$. We remark that $W_{\delta, r}^{1}=\emptyset$ in the case when $n=3$. Since $\beta_{\delta}^{1}\left(S_{\delta, r}\right)=T_{\delta, r} \cap P$ is the fiber of the composition

$$
T_{\delta, r} \subset J(C) \stackrel{N}{\longrightarrow} J(E)
$$

at $0 \in J(E)$, we have $\operatorname{dim} \beta_{\delta}^{1}\left(S_{\delta, r}\right)=n-3$. Let $T_{\delta, 2 r} \subset T_{\delta, r}$ be the image of the morphism

$$
\begin{aligned}
C^{(n-4)} \times E & \longrightarrow \operatorname{Pic}^{0}(C)=J(C), \\
(\mathbf{q}, \phi(q)) & \longmapsto \mathcal{O}_{C}(\mathbf{q}+2 r+q+\sigma(q)) \otimes \delta^{\vee} .
\end{aligned}
$$

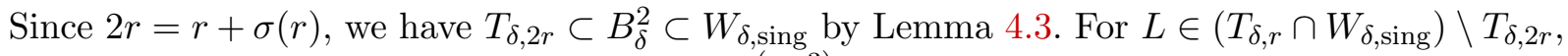
there is an element $\left(q_{1}+\cdots+q_{n-3}, \phi(q)\right) \in C^{(n-3)} \times E$ such that $L=\mathcal{O}_{C}\left(q_{1}+\cdots+q_{n-3}+r+\right.$ $q+\sigma(q)) \otimes \delta^{\vee}$ and $r \notin\left\{q_{1}, \ldots, q_{n-3}\right\}$. If

$$
q_{1}^{\prime}+\cdots+q_{n-i}^{\prime}+i r \in\left|\Omega_{C}^{1}\left(-q_{1}-\cdots-q_{n-3}-r-q-\sigma(q)\right)\right|=\left|\Omega_{C}^{1} \otimes(L \otimes \delta)^{\vee}\right|
$$

and $r \notin\left\{q_{1}^{\prime}, \ldots, q_{n-i}^{\prime}\right\}$, then by Lemma 5.2, the number $i$ is odd. In the same way, any member in the linear system $\left|\Omega_{C}^{1}\left(-q_{1}^{\prime}-\cdots-q_{n-i}^{\prime}-i r\right)\right|=|L \otimes \delta|$ has an odd multiplicity at $r$. This implies that $h^{0}\left(C, L \otimes \mathcal{O}_{C}(-r) \otimes \delta\right)=h^{0}(C, L \otimes \delta)>1$. Hence we have

$$
T_{\delta, r} \cap W_{\delta, \text { sing }}=T_{\delta, 2 r} \cup\left(T_{\delta, r} \cap W_{\delta, r}^{1}\right) .
$$

When $n=3$, this implies that $T_{\delta, r} \cap W_{\delta \text {,sing }}=\emptyset$. When $n \geqslant 4$,

$$
\beta_{\delta}^{1}\left(S_{\delta, r}\right) \cap W_{\delta, \text { sing }}=\left(T_{\delta, 2 r} \cap P\right) \cup\left(\beta_{\delta}^{1}\left(S_{\delta, r}\right) \cap W_{\delta, r}^{1}\right)
$$

is a proper closed subset of $\beta_{\delta}^{1}\left(S_{\delta, r}\right)=T_{\delta, r} \cap P$ because $\operatorname{dim}\left(T_{\delta, 2 r} \cap P\right) \leqslant n-4$ and $\operatorname{dim} W_{\delta, r}^{1} \leqslant$ $n-4$.

Let $Z_{\delta, r}=\psi_{\delta}\left(S_{\delta, r}\right)$ be the image of $S_{\delta, r}$ by $\psi_{\delta}: Y_{\delta} \rightarrow X_{\delta}$. Then

$$
Z_{\delta, r}=\left\{(\mathbf{p}, p) \in X_{\delta} \mid \mathbf{p} \geqslant \phi(r)\right\} .
$$

Lemma 5.16. If $n \geqslant 4$, then $Z_{\delta, r}$ is irreducible of dimension $n-3$. If $n=3$, then $X_{\delta} \cong E$ and $Z_{\delta, r} \subset X_{\delta}$ is a $J\left(X_{\delta}\right)_{2}$-orbit by the natural action of $J\left(X_{\delta}\right)$ on the curve $X_{\delta}$ of genus 1 . 


\section{A. IKEDA}

Proof. Let $\gamma_{\delta, p_{0}}: E^{(n-2)} \rightarrow J(E)$ be the morphism given in the proof of Lemma 5.9 for fixed $p_{0} \in E$, and let $i_{r}: E^{(n-3)} \rightarrow E^{(n-2)}$ be the morphism defined by

$$
i_{r}: E^{(n-3)} \longrightarrow E^{(n-2)}, \quad \mathbf{p} \longmapsto \mathbf{p}+\phi(r) .
$$

If $n \geqslant 4$, then $Z_{\delta, r}$ is a $\mathbf{P}^{n-4}$-bundle over $E$ by the base change

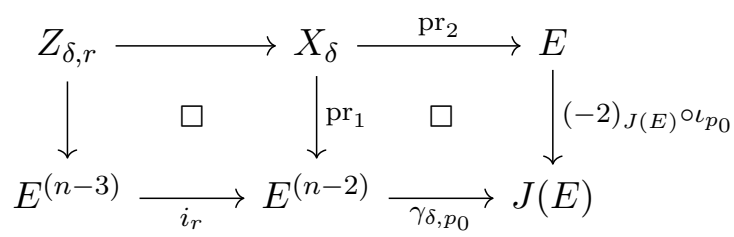

of the $\mathbf{P}^{n-4}$-bundle $\gamma_{\delta, p_{0}} \circ i_{r}: E^{(n-3)} \rightarrow J(E)$; hence $Z_{\delta, r}$ is irreducible of dimension $n-3$. If $n=3$, then $\mathrm{pr}_{2}: X_{\delta} \rightarrow E$ is an isomorphism and

$$
Z_{\delta, r} \cong\left\{p \in E \mid \mathcal{O}_{E}(\phi(r)+2 p) \cong N(\delta)\right\}
$$

is an orbit by the $J(E)_{2}$-action.

We denote the ramification divisor of $\psi_{\delta}^{\circ}=\left.\psi_{\delta}\right|_{Y_{\delta}^{\circ}}: Y_{\delta}^{\circ} \rightarrow X_{\delta}$ by $\operatorname{Ram}\left(\psi_{\delta}^{\circ}\right) \subset Y_{\delta}^{\circ}$.

Lemma 5.17. We have

$$
\overline{\psi_{\delta}^{\circ}\left(\operatorname{Ram}\left(\psi_{\delta}^{\circ}\right)\right)}=\bigcup_{r \in \operatorname{Ram}(\phi)} Z_{\delta, r} .
$$

Proof. Since $\operatorname{Ram}\left(\psi_{\delta}\right)=R_{\delta} \cup \bigcup_{r \in \operatorname{Ram}(\phi)} S_{\delta, r}$, by Lemma 5.14 we have the equality $\operatorname{Ram}\left(\psi_{\delta}^{\circ}\right)=$ $\bigcup_{r \in \operatorname{Ram}(\phi)} S_{\delta, r} \cap Y_{\delta}^{\circ}$. By Lemmas 5.5 and 5.15, we have $S_{\delta, r} \cap Y_{\delta}^{\circ} \neq \emptyset$ for $n \geqslant 3$ and $S_{\delta, r} \cap Y_{\delta}^{\circ}=S_{\delta, r}$ for $n=3$. Since $\psi_{\delta}$ is a finite morphism, $\psi_{\delta}^{\circ}\left(S_{\delta, r} \cap Y_{\delta}^{\circ}\right)$ is of dimension $n-3$. By Lemma 5.16, we have $\overline{\psi_{\delta}^{\circ}\left(S_{\delta, r} \cap Y_{\delta}^{\circ}\right)}=\psi_{\delta}\left(S_{\delta, r}\right)=Z_{\delta, r}$.

Let $H_{r} \subset \mathbf{P}\left(H^{0}(E, \eta)^{\vee}\right)$ be the hyperplane corresponding to the subspace

$$
H^{0}\left(E, \eta \otimes \mathcal{O}_{E}(-\phi(r))\right) \subset H^{0}(E, \eta) .
$$

LEMmA 5.18. The hyperplane $H_{r}$ is the unique hyperplane with the property $\nu_{\delta}\left(Z_{\delta, r}\right) \subset H_{r}$.

Proof. The inclusion $\nu_{\delta}\left(Z_{\delta, r}\right) \subset H_{r}$ is obvious. We prove the uniqueness of the hyperplane $H_{r}$. Let $z=\left(p_{1}+\cdots+p_{n-3}+\phi(r), p\right) \in Z_{\delta, r}$ satisfy $p_{i} \neq t_{\eta-N(\delta)}\left(p_{j}\right)$ for $i \neq j$. We take a point $p^{\prime} \in E \backslash\{p\}$ such that $\mathcal{O}_{E}\left(2 p^{\prime}\right) \cong \mathcal{O}_{E}(2 p)$ and $\mathcal{O}_{E}\left(p^{\prime}-p\right) \nsubseteq \eta \otimes(N(\delta))^{\vee}$. Then $z^{\prime}=\left(p_{1}+\cdots+p_{n-3}+\phi(r), p^{\prime}\right)$ is contained in $Z_{\delta, r}$, and $\nu_{\delta}(z) \neq \nu_{\delta}\left(z^{\prime}\right)$. This implies the uniqueness in the case when $n=3$. When $n \geqslant 4$, we show that $\nu_{\delta}\left(Z_{\delta, r}\right) \subset H_{r}$ is a nonlinear hypersurface in $H_{r}$. Let $l \subset H_{r}$ be the line containing the two points $\nu_{\delta}(z), \nu_{\delta}\left(z^{\prime}\right) \in \nu_{\delta}\left(Z_{\delta, r}\right)$. Then the line $l \subset \mathbf{P}\left(H^{0}(E, \eta)^{\vee}\right)$ corresponds to the linear pencil

$$
\left|\eta\left(-p_{1}-\cdots-p_{n-3}-\phi(r)\right)\right| \subset|\eta| \cong \mathbf{P}\left(H^{0}(E, \eta)^{\vee}\right) .
$$

For a point $p_{0} \in E$, there is a unique point $p_{0}^{\prime} \in E$ such that $p_{0}+p_{0}^{\prime} \in\left|\eta\left(-p_{1}-\cdots-p_{n-3}-\phi(r)\right)\right|$. If $\mathcal{O}_{E}\left(2 p_{0}\right) \varsubsetneqq \mathcal{O}_{E}(2 p), \mathcal{O}_{E}\left(2 p_{0}^{\prime}\right) \varsubsetneqq \mathcal{O}_{E}(2 p)$, and

$$
p_{0}, p_{0}^{\prime} \notin\left\{t_{\eta-N(\delta)}\left(p_{1}\right), \ldots, t_{\eta-N(\delta)}\left(p_{n-3}\right), t_{N(\delta)-\eta}\left(p_{1}\right), \ldots, t_{N(\delta)-\eta}\left(p_{n-3}\right)\right\},
$$

then the point $p_{1}+\cdots+p_{n-3}+\phi(r)+p_{0}+p_{0}^{\prime} \in|\eta|$ on the line $l$ is not contained in $\nu_{\delta}\left(Z_{\delta, r}\right)$.

Lemma 5.19. The pull-back of the divisor $H_{r}$ on $\mathbf{P}\left(H^{0}(E, \eta)^{\vee}\right)$ by $\nu_{\delta}: X_{\delta} \rightarrow \mathbf{P}\left(H^{0}(E, \eta)^{\vee}\right)$ is

$$
\nu_{\delta}^{*} H_{r}=Z_{\delta, r}+M_{\delta, r}+M_{\delta, r}^{\prime},
$$




\section{PRYM-TORELLI FOR COVERINGS OF ELLIPTIC CURVES}

where $M_{\delta, y}$ and $M_{\delta, y}^{\prime}$ are the irreducible divisors on $X_{\delta}$ defined by

$$
\begin{aligned}
& M_{\delta, r}=\left\{(\mathbf{p}, p) \in X_{\delta} \mid p=\phi(r)\right\}, \\
& M_{\delta, r}^{\prime}=\left\{(\mathbf{p}, p) \in X_{\delta} \mid p=t_{N(\delta)-\eta}(\phi(r))\right\} .
\end{aligned}
$$

Proof. Let $I_{r}$ be the irreducible divisor on $E^{(n)}$ defined by

$$
I_{r}=\left\{\mathbf{p} \in E^{(n)} \mid \mathbf{p} \geqslant \phi(r)\right\},
$$

and let $Z_{r}, M_{r}, M_{r}^{\prime}$ be the irreducible divisors on $E^{(n-2)} \times E$ defined by

$$
\begin{aligned}
& Z_{r}=\left\{(\mathbf{p}, p) \in E^{(n-2)} \times E \mid \mathbf{p} \geqslant \phi(r)\right\}, \\
& M_{r}=\left\{(\mathbf{p}, p) \in E^{(n-2)} \times E \mid p=\phi(r)\right\}, \\
& M_{r}^{\prime}=\left\{(\mathbf{p}, p) \in E^{(n-2)} \times E \mid p=t_{N(\delta)-\eta}(\phi(r))\right\} .
\end{aligned}
$$

Then the pull-back of the divisor $I_{r}$ by the morphism

$$
\begin{aligned}
E^{(n-2)} \times E & \longrightarrow E^{(n)}, \\
(\mathbf{p}, p) & \longmapsto \mathbf{p}+p+t_{\eta-N(\delta)}(p)
\end{aligned}
$$

is the divisor $Z_{r}+M_{r}+M_{r}^{\prime}$ on $E^{(n-2)} \times E$. Since the restriction of $I_{r}$ to $|\eta| \subset E^{(n)}$ is the divisor $H_{r}$ on $\mathbf{P}\left(H^{0}(E, \eta)^{\vee}\right) \cong|\eta|$, the pull-back $\nu_{\delta}^{*} H_{r}$ is the restriction of $Z_{r}+M_{r}+M_{r}^{\prime}$ to $X_{\delta}$.

Corollary 5.20. The divisor $\nu_{\delta}^{*} H_{r}-Z_{\delta, r}$ on $X_{\delta}$ is irreducible if and only if $N(\delta)=\eta$.

We consider the dual variety $\left(\Phi_{|\eta|}(E)\right)^{\vee} \subset \mathbf{P}\left(H^{0}(E, \eta)^{\vee}\right)$ of the image of the closed immersion $\Phi_{|\eta|}: E \rightarrow \mathbf{P}\left(H^{0}(E, \eta)\right)$ which is defined by the linear system $|\eta|$.

Lemma 5.21. The projective curve $\Phi_{|\eta|}(E) \subset \mathbf{P}\left(H^{0}(E, \eta)\right)$ is reflexive. In particular, $\Phi_{|\eta|}(E)=$ $\left(\left(\Phi_{|\eta|}(E)\right)^{\vee}\right)^{\vee} \subset \mathbf{P}\left(H^{0}(E, \eta)\right)$.

Proof. If $1 \leqslant i<n$, then $h^{0}\left(E, \eta \otimes \mathcal{O}_{E}(-i p)\right)=n-i$ for any $p \in E$. If $n=3$, then $h^{0}(E, \eta \otimes$ $\left.\mathcal{O}_{E}(-3 p)\right)=0$ for general $p \in E$. Hence $h^{0}\left(E, \eta \otimes \mathcal{O}_{E}(-2 p)\right)>h^{0}\left(E, \eta \otimes \mathcal{O}_{E}(-3 p)\right)$ for general $p \in E$. Then there is a hyperplane $H \subset \mathbf{P}\left(H^{0}(E, \eta)\right)$ which intersects $\Phi_{|\eta|}(E)$ at $\Phi_{|\eta|}(p)$ with the multiplicity 2. By [HK85, (3.5)], the projective curve $\Phi_{|\eta|}(E) \subset \mathbf{P}\left(H^{0}(E, \eta)\right)$ is reflexive because the characteristic of the base field $k$ is not equal to 2 .

Lemma 5.22. If $N(\delta)=\eta$, then the dual variety of $X_{\delta}^{\prime} \subset \mathbf{P}\left(H^{0}(E, \eta)^{\vee}\right)$ is $\Phi_{|\eta|}(E) \subset \mathbf{P}\left(H^{0}(E, \eta)\right)$.

Proof. By Lemma 5.21, we show that the dual variety of $\Phi_{|\eta|}(E)$ is $X_{\delta}^{\prime}$. For $L \in\left(B_{\delta} \cap P\right) \backslash D_{\delta \text {,sing }}$ $\subset \operatorname{Pic}^{0}(C)$, there is a unique effective divisor $\mathbf{q}+q+\sigma(q) \in C^{(n)}$ such that $L \otimes \delta \cong \mathcal{O}_{C}(\mathbf{q}+q+\sigma(q))$. Since $L \in P$, we have

$$
\eta=N(\delta)=\left[\mathcal{O}_{E}\left(\phi^{(n-2)}(\mathbf{q})+2 \phi(q)\right)\right] \in \operatorname{Pic}^{n}(E),
$$

hence

$$
\Omega_{C}^{1} \otimes L^{\vee} \otimes \delta^{\vee} \cong \phi^{*} \eta \otimes L^{\vee} \otimes \delta^{\vee} \cong \mathcal{O}_{C}(\sigma(\mathbf{q})+\sigma(q)+q),
$$

and $\Psi_{J(C), \delta}^{B}(L) \in \mathbf{P}\left(H^{0}(E, \eta)^{\vee}\right)$ is defined by the effective divisor

$$
\phi^{(n-2)}(\mathbf{q})+2 \phi(q) \in|\eta| \cong \mathbf{P}\left(H^{0}(E, \eta)^{\vee}\right) .
$$

This means that the hyperplane in $\mathbf{P}\left(H^{0}(E, \eta)\right)$ corresponding to $\Psi_{J(C), \delta}^{B}(L)$ is tangent to the image $\Phi_{|\eta|}(E)$. Hence we have $\Psi_{J(C), \delta}^{B}\left(\left(B_{\delta} \cap P\right) \backslash D_{\delta \text {,sing }}\right) \subset\left(\Phi_{|\eta|}(E)\right)^{\vee}$. Since $\left(\Phi_{|\eta|}(E)\right)^{\vee}$ and $\Psi_{J(C), \delta}^{B}\left(\left(B_{\delta} \cap P\right) \backslash D_{\delta \text {,sing }}\right)$ are irreducible hypersurfaces in $\mathbf{P}\left(H^{0}(E, \eta)^{\vee}\right)$, we have $X_{\delta}^{\prime}=\left(\Phi_{|\eta|}(E)\right)^{\vee}$. 


\section{A. IKEDA}

\section{Key propositions}

Let $\mathcal{L}$ be an ample invertible sheaf on $P$ which represents the polarization $\lambda_{P}$.

Lemma 6.1. The variety $U_{D}=\mathrm{Bs}|\mathcal{L}| \backslash D_{\text {sing }}$ is nonsingular for any $D \in|\mathcal{L}|$.

Proof. Since $D=D_{\delta}$ for some $\delta \in \operatorname{Pic}^{n}(C)$, this is a consequence of Lemmas 4.6 and 5.10.

Consider the Gauss map

$$
\Psi_{D}: D \backslash D_{\text {sing }} \longrightarrow \mathbf{P}^{n-1}=\operatorname{Grass}\left(n-1, H^{0}\left(P, \Omega_{P}^{1}\right)^{\vee}\right)
$$

for $D \in|\mathcal{L}|$, and let $\nu_{D}: X_{D} \rightarrow X_{D}^{\prime}$ be the normalization of $X_{D}^{\prime}=\overline{\Psi_{D}\left(U_{D}\right)} \subset \mathbf{P}^{n-1}$. Then by Lemma 6.1 , there is a unique morphism $\psi_{D}: U_{D} \rightarrow X_{D}$ such that $\left.\Psi_{D}\right|_{U_{D}}=\nu_{D} \circ \psi_{D}$. Let $Z_{D}=\overline{\psi_{D}\left(\operatorname{Ram}\left(\psi_{D}\right)\right)} \subset X_{D}$ be the Zariski closure of the image of the ramification divisor of $\psi_{D}$.

Proposition 6.2. Let $D \subset P$ be a member in $|\mathcal{L}| \backslash \Pi_{\mathcal{L}}$, where $\Pi_{\mathcal{L}} \subset|\mathcal{L}|$ is the subset in Lemma 4.8.

(i) If $n=3$, then $X_{D}$ is a nonsingular projective curve of genus 1 and $Z_{D}$ is a disjoint union of six orbits $Z_{D, 1}, \ldots, Z_{D, 6}$ by the $J\left(X_{D}\right)_{2}$-action.

(ii) If $n \geqslant 4$, then $Z_{D}$ has $2 n$ irreducible components $Z_{D, 1}, \ldots, Z_{D, 2 n}$.

(iii) For any subset $Z_{D, i} \subset Z_{D}$ in parts (i) and (ii), there is a unique hyperplane $H_{D, i} \subset \mathbf{P}^{n-1}$ such that $\nu_{D}\left(Z_{D, i}\right) \subset H_{D, i}$.

Proof. By Lemma 4.7, there is a $\delta \in \operatorname{Pic}^{n}(C)$ such that $N(\delta)=\eta$ and $\mathcal{L} \cong \mathcal{L}_{\delta}$. By Lemma 4.6, there is an $s \in \operatorname{Pic}^{0}(E)$ such that $D=D_{\delta+\phi^{*} s}$. By the proof of Lemma 4.8, we have that $D \notin \Pi_{\mathcal{L}}$ implies $s \notin J(E)_{4} \backslash J(E)_{2}$. By Lemma 5.7, the Gauss map $\left.\Psi_{D}\right|_{U_{D}}: U_{D} \rightarrow \mathbf{P}^{n-1}$ is identified with $\left.\Psi_{J(C), \delta+\phi^{*} s}^{B}\right|_{U_{D}}: U_{D} \rightarrow \mathbf{P}\left(H^{0}(E, \eta)^{\vee}\right)$. Since $N\left(\delta+\phi^{*} s\right)-\eta=2 s \notin J(E)_{2} \backslash\{0\}$, by Lemma 5.12, the normalization of $X_{\delta+\phi^{*} s}^{\prime}=X_{D}^{\prime}$ is given by $\nu_{\delta+\phi^{*} s}: X_{\delta+\phi^{*} s} \rightarrow X_{\delta+\phi^{*} s}^{\prime}$, and by Lemmas 5.8 and 5.10, the map $\psi_{D}: U_{D} \rightarrow X_{D}$ is identified with $\psi_{\delta+\phi^{*} s}^{\circ}: Y_{\delta+\phi^{*} s}^{\circ} \rightarrow X_{\delta+\phi^{*} s}$. Hence the statements (i), (ii), and (iii) are consequences of Lemmas 5.16, 5.17, and 5.18.

We define the subset $\Pi_{\mathcal{L}}^{\prime}$ in the linear pencil $|\mathcal{L}|$ by

$$
\Pi_{\mathcal{L}}^{\prime}=\left\{D \in|\mathcal{L}| \backslash \Pi_{\mathcal{L}} \mid \nu_{D}^{*} H_{D, i}-Z_{D, i} \text { is irreducible for } 1 \leqslant i \leqslant 2 n\right\} .
$$

Lemma 6.3. We have $\# \Pi_{\mathcal{L}}^{\prime}=4$.

Proof. We use the same identification for Gauss maps as in the proof of Proposition 6.2. Then by Corollary 5.20,

$$
D=D_{\delta+\phi^{*} s} \in \Pi_{\mathcal{L}}^{\prime} \Longleftrightarrow N\left(\delta+\phi^{*} s\right)=\eta \Longleftrightarrow s \in J(E)_{2},
$$

and by Lemma 4.4, we have $\# \Pi_{\mathcal{L}}^{\prime}=\# J(C)_{2}=4$.

Let $e_{1}+\cdots+e_{2 n}$ be the branch divisor of the original covering $\phi: C \rightarrow E$, and let $\eta \in \operatorname{Pic}(E)$ be the invertible sheaf with $\phi^{*} \eta \cong \Omega_{C}^{1}$.

Proposition 6.4. For any member $D \in \Pi_{\mathcal{L}}^{\prime}$, there is an isomorphism

$$
\left(E, e_{1}+\cdots+e_{2 n}, \eta\right) \cong\left(\left(X_{D}^{\prime}\right)^{\vee}, H_{D, 1}^{\vee}+\cdots+H_{D, 2 n}^{\vee},\left.\mathcal{O}_{\left(\mathbf{P}^{n-1}\right) \vee}(1)\right|_{\left(X_{D}^{\prime}\right)^{\vee}}\right),
$$

where $H_{D, i}^{\vee} \in\left(\mathbf{P}^{n-1}\right)^{\vee}$ is the point corresponding to the hyperplane $H_{D, i}$ and $\left(X_{D}^{\prime}\right)^{\vee} \subset\left(\mathbf{P}^{n-1}\right)^{\vee}$ is the dual variety of $X_{D}^{\prime} \subset \mathbf{P}^{n-1}$. 


\section{PRYM-TORELli FOR COVERINGS OF ELLIPTIC CURVES}

Proof. We use the same identification for Gauss maps as in the proof of Proposition 6.2. When $D \in \Pi_{\mathcal{L}}^{\prime}$, we may assume $D=D_{\delta}$ and $N(\delta)=\eta$ by Corollary 5.20. Then the point $H_{D, i}^{\vee}$ is identified with the point $H_{r}^{\vee}=\Phi_{|\eta|}(\phi(r))$ for $r \in \operatorname{Ram}(\phi)$, and $\left(X_{D}^{\prime}\right)^{\vee}$ is identified with $\left(X_{\delta}^{\prime}\right)^{\vee}$, which coincides with $\Phi_{|\eta|}(E) \subset \mathbf{P}\left(H^{0}(E, \eta)\right)$ by Lemma 5.22 .

Remark 6.5. For a member $D \in \Pi_{\mathcal{L}}^{\prime}$, the Gauss map $\Psi_{D}: D \backslash D_{\text {sing }} \rightarrow \mathbf{P}^{n-1}$ is a covering of degree $2^{n}$, and $X_{D}^{\prime} \cup \bigcup_{i=1}^{2 n} H_{D, i}$ is the branch locus of $\Psi_{D}$. But for $D \notin \Pi_{\mathcal{L}}^{\prime}$, the Gauss map $\Psi_{D}$ is not easy to compute.

\section{ACKNOWLEDGEMENTS}

The author would like to thank Juan Carlos Naranjo for informing him of the recent paper [NO19].

\section{REFERENCES}

ACGH85 E. Arbarello, M. Cornalba, P. A. Griffiths, and J. Harris, Geometry of algebraic curves, Vol. I, Grundlehren math. Wiss., vol. 267 (Springer-Verlag, New York, 1985); doi:10.1007/978-14757-5323-3.

AM67 A. Andreotti and A. L. Mayer, On period relations for abelian integrals on algebraic curves, Ann. Scuola Norm. Sup. Pisa Cl. Sci. (3) 21 (1967), 189-238; http://www.numdam.org/item/?id= ASNSP_1967_3_21_2_189_0.

And58 A. Andreotti, On a theorem of Torelli, Amer. J. Math. 80 (1958), 801-828; doi:10.2307/ 2372835.

BCV95 F. Bardelli, C. Ciliberto, and A. Verra, Curves of minimal genus on a general abelian variety, Compos. Math. 96 (1995), no. 2, 115-147; http://www.numdam.org/item?id=CM_1995__96_2_ 115_0.

Don81 R. Donagi, The tetragonal construction, Bull. Amer. Math. Soc. 4 (1981), no. 2, 181-185; doi:10.1090/S0273-0979-1981-14875-8.

DS81 R. Donagi and R. Smith, The structure of the Prym map, Acta Math. 146 (1981), no. 1-2, 25-102; doi:10.1007/BF02392458.

FS82 R. Friedman and R. Smith, The generic Torelli theorem for the Prym map, Invent. Math. 67 (1982), no. 3, 473-490; doi:10.1007/BF01398932.

HK85 A. Hefez and S.L. Kleiman, Notes on the duality of projective varieties, Geometry Today (Rome, 1984), Progr. Math., vol. 60 (Birkhäuser Boston, Boston, MA, 1985), 143-183.

Kan83 V.I. Kanev, A global Torelli theorem for Prym varieties at a general point, Math. USSR Izv. 20 (1983), no. 2, 235-257; doi:10.1070/IM1983v020n02ABEH001349.

Mar67 H. H. Martens, On the varieties of special divisors on a curve, J. reine angew. Math. 227 (1967), 111-120; doi:10.1515/crll.1967.227.111.

Mil86 J. S. Milne, Jacobian varieties, Arithmetic Geometry (Storrs, Conn., 1984) (eds G. Cornell and J. H. Silverman; Springer, New York, 1986), 167-212; doi :10.1007/978-1-4613-8655-1_7.

MN14 V.O. Marcucci and J.C. Naranjo, Prym varieties of double coverings of elliptic curves, Int. Math. Res. Not. 2014 (2014), no. 6, 1689-1698; doi:10.1093/imrn/rns271.

MP12 V. O. Marcucci and G. P. Pirola, Generic Torelli theorem for Prym varieties of ramified coverings, Compos. Math. 148 (2012), no. 4, 1147-1170; doi:10.1112/S0010437X12000280.

Mum74 D. Mumford, Prym varieties. I, Contributions to Analysis (a Collection of Papers Dedicated to Lipman Bers) (Academic Press, New York, 1974), 325-350.

Nar92 J. C. Naranjo, Prym varieties of bi-elliptic curves, J. reine angew. Math. 424 (1992), 47-106; doi:10.1515/crll.1992.424.47. 


\section{A. IKEDA}

NO19 J. C. Naranjo and A. Ortega, Generic injectivity of the Prym map for double ramified coverings (with an appendix by Alessandro Verra), Trans. Amer. Math. Soc. 371 (2019), no. 5, 3627-3646; doi:10.1090/tran/7459.

NR95 D.S. Nagaraj and S. Ramanan, Polarisations of type $(1,2, \ldots, 2)$ on abelian varieties, Duke Math. J. 80 (1995), no. 1, 157-194; doi:10.1215/S0012-7094-95-08007-7.

Ver04 A. Verra, The Prym map has degree two on plane sextics, The Fano Conference (University Torino, Turin, 2004), 735-759.

Atsushi Ikeda atsushi@mail.dendai.ac.jp

Department of Mathematics, School of Engineering, Tokyo Denki University, Adachi-ku, Tokyo 120-8551, Japan 